\title{
ARTÍCULOS
}

\section{Dinámica geomorfológica fluvial y cambios de usos del suelo: impacto en los reservorios de carbono de suelos y sedimentos}

\author{
Fluvial geomorphological dynamics and land use changes: \\ impact on the organic carbon stocks of soil and sediment
}

\author{
Amar Halifa-Marín ${ }^{\mathrm{b}}$, Pedro Pérez-Cutillas ${ }^{\mathrm{a}, \mathrm{b} *}$, María Almagro ${ }^{\mathrm{b}, \mathrm{c}}$, \\ María Martínez-Mena ${ }^{\text {b }}$, Carolina Boix-Fayos ${ }^{\text {b }}$ \\ ${ }^{a}$ Universidad de Murcia, Facultad de Letras, Departamento de Geografía, Murcia, España. \\ *Autor de correspondencia: ${ }^{\text {b }}$ CEBAS-CSIC, Departamento de Conservación de Suelos y Agua y Manejo de Residuos Orgánicos, \\ Murcia, España, teléfono: +34 968 396200, Ext. 6256, perezcutillas@cebas.csic \\ c BC3-Basque Centre for Climate Change, Sede Building 1, 1st floor, \\ Scientific Campus of the University of the Basque Country, 48940, Leioa, Basque Country, Spain.
}

\section{SUMMARY}

The drainage basin of the Turrilla river (SE of Spain) went through important land cover changes since 1950s; from mainly an agrarian scenario in 1956 to other depopulated and forested in 2015. This study analyzes the effects of land use changes on fluvial dynamics and their relationship with the organic carbon (OC) stock in fluvial sedimentary deposits as well as in the soil of the basin. Methods included a fluvial geomorphological analysis and a land use change analysis in combination with OC databases of soil and sediment. Results showed that the fluvial channel experienced important morphological changes related to different erosion processes and stabilization of fluvial deposits, induced by land use changes in the drainage area. The active channel decreased $63 \%$ in the study period, while bank erosion and gully erosion increased (34\% and $21 \%$, respectively). Alluvial fans and alluvial plain were also extended ( $21 \%$ and $7 \%$, respectively) and alluvial bars were colonized by vegetation. Sediment was impoverished in OC compared to catchment soils $\left(0.24\right.$ enrichment ratio sediment/soil). However the increase of OC stock $\left(\mathrm{Mg} \mathrm{ha}^{-1}\right)$ was very similar between soil $(25 \%)$ and sediment $(23 \%)$. The total reservoir of OC $(\mathrm{Mg})$ increased $27 \%$ in sediments and $25 \%$ in the catchment soils. Results show the large influence of geomorphological dynamics on the OC reservoir at the catchment scale. A very high potential of fluvial sediments to increase OC sinks was observed, particularly in scenarios where the active channel is narrowed and the fluvial channel is encroached with vegetation, facilitating the input of OC in sediment. The potential of sediment to sequester organic carbon could be very useful in planning and management of fluvial sedimentary zones in climate change mitigation policies.

Key words: sediment management, OC reservoirs, OC stock, organic carbon, land use change, sedimentary deposits.

\section{RESUMEN}

Este estudio analizó los efectos de los cambios de uso del suelo en la dinámica fluvial y su relación con el reservorio de carbono orgánico (CO) en los depósitos de sedimentos fluviales, así como en los suelos de la cuenca. Para ello se utilizó una combinación de cartografía geomorfológica fluvial y de los usos del suelo, con bases de datos de diferentes muestreos de CO en suelos y sedimentos. La superficie del cauce se redujo (-63\%) en el período de estudio, al tiempo que se ampliaron la erosión lateral (34 \%) y en cárcavas (21\%), los abanicos aluviales (13\%) y la llanura aluvial (7\%). Las barras fluviales fueron colonizadas en un porcentaje muy elevado por la vegetación. Los sedimentos se encontraron empobrecidos en CO con respecto a los suelos de la cuenca ( 0,24 razón de enriquecimiento de CO sedimento/suelo). Sin embargo, el aumento de las existencias (stock) de CO ( $\mathrm{Mg}^{-1}$ ) fue muy similar en el período de estudio en suelos $(25 \%)$ y en sedimentos (23\%). Así como el reservorio total de $\mathrm{CO}(\mathrm{Mg})$ que aumentó un $27 \%$ en los sedimentos del cauce y un $25 \%$ en los suelos de la cuenca. Los resultados mostraron la gran influencia de la dinámica geomorfológica en los reservorios de $\mathrm{CO}$ a escala de cuenca. Los sedimentos fluviales poseen gran potencial para generar sumideros de CO, en escenarios de estrechamiento del cauce activo y reverdecimiento del lecho, facilitando la entrada de CO.

Palabras clave: carbono orgánico, reservorios de carbono orgánico, existencias de carbono orgánico, cambios en el uso del suelo, depósitos de sedimentos. 


\section{INTRODUCCIÓN}

Los cambios de uso del suelo en las cuencas de drenaje pueden alterar el balance de sedimentos y caudal (Kondolf et al. 2002, Stott y Mount 2004), siendo muchas cuencas hidrológicas mediterráneas de Europa un buen ejemplo de ello (García-Ruíz y Lana-Renault 2011). En España, la despoblación y el desarrollo de las políticas para la restauración de la cubierta vegetal, el control de la erosión y la prevención de avenidas permitieron que la cobertura vegetal aumentase enormemente en numerosas cuencas de montaña durante las últimas décadas (Vadell et al. 2016). Como consecuencia de la recuperación de la cubierta vegetal pueden ocurrir cambios en el balance del ciclo hidrológico, pues una mayor densidad de la cobertura vegetal implica también un mayor consumo de agua, además del incremento de las pérdidas por evapotranspiración (PérezCutillas et al. 2018). Ligado a los efectos en el ciclo hidrológico, la recuperación de la cubierta vegetal tiene un efecto determinante en la dinámica sedimentaria, todo ello ha sido objeto de estudio tanto en la cuenca mediterránea (Boix-Fayos et al. 2007, García-Ruíz y Lana-Renault 2011, Pérez-Cutillas et al. 2018), como en otras regiones del planeta (Kondolf et al. 2002, Stott y Mount 2004, Sun-Kim et al. 2017, Wei-Kuo et al. 2017). En ese escenario ambiental, con una cobertura vegetal más extensa, se observó que los suelos se mostraron más resistentes a la erosión y permeables, y, por tanto, el flujo de sedimentos y agua movilizados en la cuenca de drenaje se redujo (Boix-Fayos et al. 2007). Con la reducción del caudal, los cauces evolucionaron, los depósitos sedimentarios se estabilizaron y se recubrieron de vegetación, al tiempo que los cauces fluviales se estrecharon y sufrieron procesos de incisión al fluir el caudal con menor carga sedimentaria (Gómez-Villar y Martínez-Castroviejo 1991, Vanacker et al. 2005).

Paralelamente, recientes investigaciones apuntan que el abandono de las áreas de montaña, con la consecuente revegetación-estabilización de las cuencas de drenaje y los sedimentos fluviales en los cauces, podría suponer un sumidero de carbono relevante en la biosfera terrestre (Wei et al. 2012, Hoffman et al. 2013, Nadeu et al. 2014, BoixFayos et al. 2015), en oposición a los estudios que apuntan las importantes emisiones de carbono como consecuencia de los procesos erosivos (Chappell et al. 2014, Ran et al. 2014, Lal et al. 2015). Sin embargo, la magnitud y los procesos relacionados con el secuestro de carbono en los sedimentos fluviales son todavía desconocidos (Hoffman et al. 2013). Un mayor conocimiento de esta cuestión, podría mejorar la gestión ambiental de los sedimentos (Lal et al. 2015) y ayudar a identificar su posible rol en el diseño de medidas de mitigación del cambio climático (Sanz-Cobena et al. 2017), tanto en los cauces como en las cuencas, de acuerdo a las recomendaciones del grupo intergubernamental de expertos sobre el cambio climático (IPCC 2014).

Este estudio se desarrolla en una cuenca de media montaña en el SE de España, representativa de muchos de los cambios socioeconómicos y medioambientales, con impacto en el paisaje fluvial, acontecidos en la franja septentrional de la cuenca mediterránea en las últimas décadas (García-Ruiz y Lana-Renault 2011). La hipótesis de este estudio propone que los cambios de uso del suelo en las cuencas de drenaje introducen alteraciones en la dinámica geomorfológica, lo que puede suponer cambios drásticos en la morfología de los cauces, y con esto, inducir cambios en los reservorios de carbono orgánico, tanto en el sedimento fluvial como en los suelos de la cuenca. Los objetivos propuestos son: i) analizar las alteraciones en la dinámica y morfología fluvial del cauce y otras formas geomorfológicas adyacentes durante el periodo 19562015; ii) analizar los cambios de la cobertura vegetal en los suelos de la cuenca y el entorno fluvial (cauce y formas geomorfológicas) durante ese periodo; y iii) estimar la evolución de los reservorios de carbono orgánico (CO) en los sedimentos fluviales y en los suelos de la cuenca de acuerdo a esas variaciones morfométricas y a los cambios en la cobertura vegetal.

\section{MÉTODOS}

Área de estudio. La cuenca hidrológica del río Turrilla se localiza en el Sureste de la Península Ibérica (figura 1). La extensión aproximada de la cuenca es $130 \mathrm{~km}^{2}$, y la longitud del cauce de estudio 19,6 km. Se trata de un curso fluvial mediterráneo, clasificado como río de montaña mediterránea calcárea (R-T12) por la Confederación Hidrográfica del Segura (CHS). Topográficamente, la cuenca presenta unos rasgos típicos de media montaña, con unos promedios de altitud y pendiente de $1.400 \mathrm{~m}$ s.n.m. y $24 \%$, respectivamente (Pérez-Cutillas et al. 2018). En cuanto al régimen climático, la temperatura anual media es $12,4^{\circ} \mathrm{C}$ (a $1.000 \mathrm{~m}$ s.n.m.), mientras que la precipitación media anual en la cuenca oscila entre los 450 y $600 \mathrm{~mm}$ (AEMET e IM 2011). Según se expone en Pérez-Cutillas et al. (2018), la textura del suelo es mayoritariamente margosa, los tipos de suelos más representativos son Cambisol Calcáreo y Regosol Calcáreo, y el análisis hidrográfico del río Turrilla muestra un cauce de orden III según Strahler, presentando una red dendrítica con una densidad de drenaje $(\mathrm{km})$ de 0,93 , y con un gradiente del cauce del 4,7\%. Tal y como se muestra en Boix-Fayos et al. (2007), se trata de un cauce altamente regulado, en el que se han podido contabilizar y estudiar el efecto geomorfológico de al menos 58 diques de corrección hidrológico forestal. Múltiples factores como la despoblación y las intervenciones estatales de corrección de la dinámica fluvial, produjeron una modificación sustancial de las coberturas del suelo en gran parte de la cuenca (Boix-Fayos et al. 2007, Pérez-Cutillas et al. 2018). En Pérez-Cutillas et al. (2018) se realiza una amplia descripción de la evolución del uso del suelo en la cuenca del Alto Taibilla desde 1956, siendo la cuenca del río Turrilla parte de la cabecera oriental. En esta área hasta mediados del S.XX el bosque denso era minoritario 


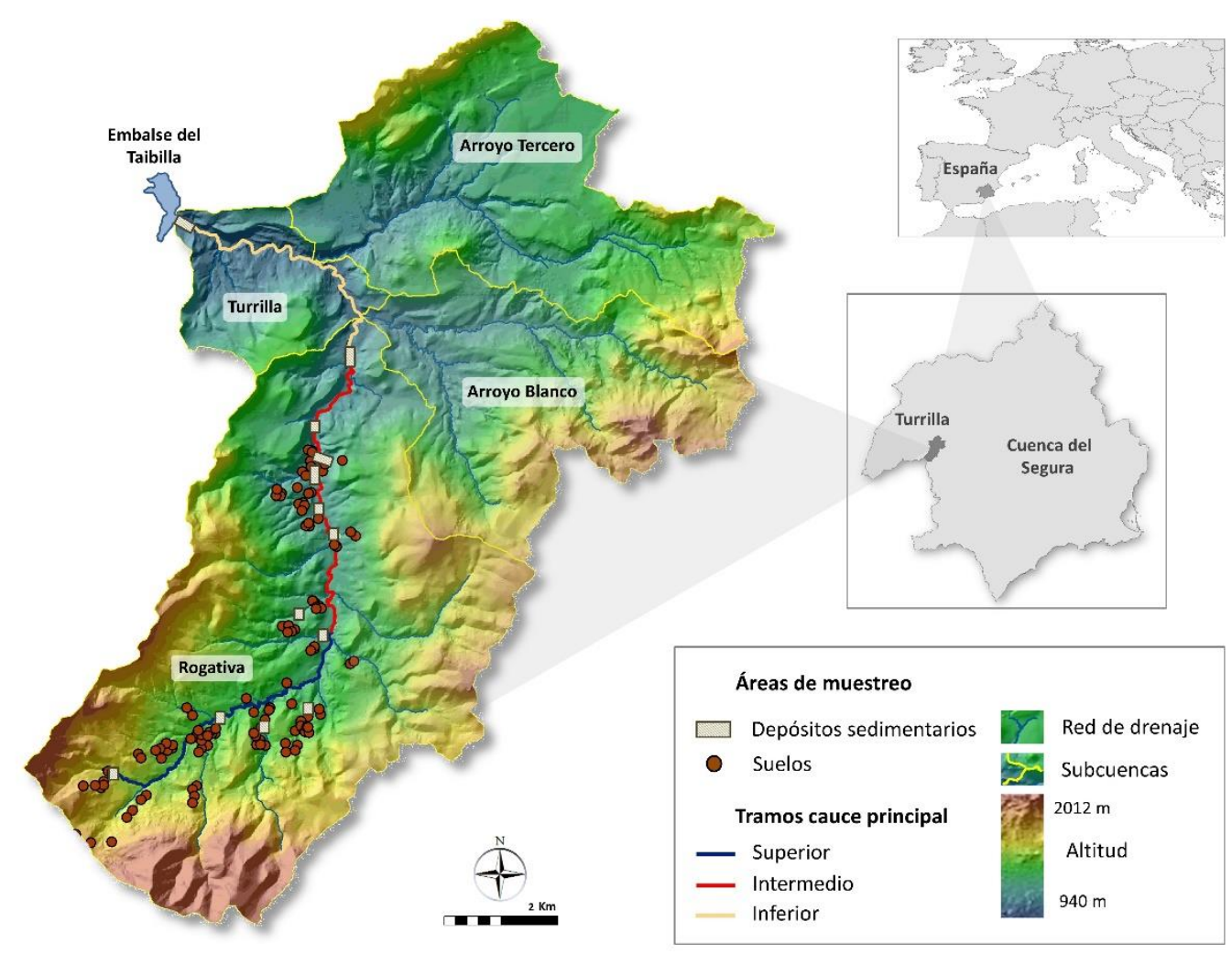

Figura 1. Área de estudio y localización de las zonas de muestreo de CO en suelos y sedimentos. La denominación Turrilla se aplica a la cuenca, aunque engloba varias subcuencas representadas en el mapa (Rogativa, Arroyo Blanco, Arroyo Tercero y Turrilla).

Study area and location of the sampling areas of soil and sediment OC. The name Turrilla is applied to the whole catchment with several subcatchments represented in the map (Rogativa, Arroyo Blanco, Arroyo Tercero and Turrilla).

respecto a la superficie del resto de usos, y predominaba el uso agrícola de secano, seguido por el bosque abierto y el matorral. Pero los cambios en los usos del suelo durante las últimas décadas provocaron un notable incremento del bosque denso, que casi triplicó su extensión entre 1956 y 2015 , mientras que el matorral y el uso agrícola disminuyeron considerablemente. Precisamente, en todas las subcuencas vertientes del río Alto Taibilla destacó el abandono de las parcelas de cultivo de secano tradicional (Pérez-Cutillas et al. 2018).

Este estudio fue abordado mediante el uso combinado de: análisis geomorfológico histórico, basado en la realización de una cartografía geomorfológica de los tramos fluviales; la recopilación de datos físico-químicos de suelos y sedimentos de trabajos anteriores; y datos de un muestreo específico. El muestreo realizado ayudó a tener una mejor representación espacial del contenido de carbono orgánico (CO) del suelo de la cuenca y los depósitos de sedimentos en el cauce, barras fluviales, y en formas geomorfológicas adyacentes al mismo.

Análisis geomorfológico. El análisis de la dinámica geomorfológica incluyó la digitalización de la morfología del cauce del río Turrilla mediante el sistema de información geográfica ArcGIS v.10.1 en el que se identificaron las barras sedimentarias dentro del cauce y se clasificaron en tres clases atendiendo a su cobertura vegetal, análisis que se expone en el siguiente apartado de métodos. Además, se identificaron en el campo y en la fotografía aérea cuatro formas geomorfológicas adyacentes al cauce, distinguiendo entre erosión lateral y erosión en cárcavas, como formas de erosión; y abanicos y llanuras aluviales, como formas sedimentarias. Las imágenes aéreas empleadas para 1956 (vuelo USAF) y para 2015 del Plan Nacional de Ortofotografía Aérea (PNOA) fueron proporcionadas por el servicio de fototeca digital del Instituto Geográfico Nacional de España (IGN). Como información auxiliar, para interpretar la topografía del cauce se empleó el modelo digital del terreno obtenido por el vuelo LIDAR, realizado en 2009, con resolución 1x1 m, proporcionado por el IGN. Por último, para establecer un procedimiento de análisis comparativo, a lo largo del curso del río se establecieron tres tramos, dividiendo el cauce en la dirección de flujo, y denominados como tramo superior, intermedio e inferior (figura 1). Para cuantificar los cambios de superficie se utilizó la tasa de variación, según la Ecuación 1: 


$$
\text { Cambio } \%=\left(\operatorname{Sup}_{\mathrm{f}}-\operatorname{Sup}_{\mathrm{i}}\right) / \operatorname{Sup}_{\mathrm{i}} * 100
$$

Donde,

Cambio $\%=$ el cambio de superficie en porcentaje.

$\operatorname{Sup}_{\mathrm{f}}=$ la superficie de la etapa final (2015).

$\operatorname{Sup}_{\mathrm{i}}=$ la superficie de la etapa inicial (1956).

Análisis de la estabilización de los sedimentos fluviales. Para relacionar los cambios geomorfológicos y el reservorio de carbono orgánico (CO) se cartografió la evolución de la vegetación en los sedimentos fluviales en el cauce y en las formas geomorfológicas adyacentes. Para analizar el cambio de la densidad vegetal en el cauce, se digitalizaron los tipos de cobertura en 1956 y en 2015 en torno a tres categorías: suelo desnudo ( $\leq 30 \%$ de cobertura vegetal), vegetación media (30-70 \%), y vegetación densa ( $\geq 70 \%$ ). Además, se utilizaron dos índices morfométricos que nos informaron de los cambios en la estabilidad del cauce: el índice braided (trenzado) según Wei-Kuo et al. (2017), y el índice de sinuosidad según Schumm (1963). El índice braided analiza si el cauce dispone de ramificaciones auxiliares, es decir, en qué medida es anastomosado, comparando la longitud del cauce principal con la longitud de flujos auxiliares en el lecho. Mientras, el índice de sinuosidad analiza el trazado del cauce, si es principalmente rectilíneo o sinuoso. Para cuantificar el cambio de la superficie para cada tipología se utilizó la tasa de variación o cambio (ecuación 1).

Cambios en el uso de los suelos en la cuenca de drena$j e$. Los cambios en la densidad vegetal de la cobertura del suelo en la cuenca de drenaje se cuantificaron mediante la realización de una cartografía específica utilizando las imágenes aéreas de 1956 y 2015 proporcionadas por el IGN, de igual forma que en el análisis geomorfológico y de la estabilización del sedimento fluvial. Esta información ya se encontraba publicada en Pérez-Cutillas et al. (2018), estudio en el que se analizaron los cambios en los usos del suelo para toda la cuenca del río Alto Taibilla, incluida la cuenca del río Turrilla. Las cartografías del uso del suelo en 1956 y 2015 en la cuenca del río Turrilla fueron revisadas de nuevo para este estudio. Nuevamente, los cambios en la distribución de la superficie de la cuenca entre las tipologías de uso se cuantificaron en valores absolutos, y en porcentaje de acuerdo a la tasa de variación (ecuación 1).

Estimación de carbono orgánico almacenado en suelos y sedimentos. Los datos del contenido total de carbono orgánico (CO) utilizados en este trabajo proceden de numerosos muestreos realizados en los distintos usos del suelo y sedimentos fluviales en la cuenca del río Turrilla publicados en trabajos anteriores (Nadeu et al. 2012, 2013, 2014, 2015, Boix-Fayos et al. 2015). Esta base de datos existente se completó con la realización de un nuevo muestreo de sedimentos en el cauce, en el que se obtuvieron cuatro puntos de muestreo adicionales en barras sedimentarias de escasa cobertura y cuatro puntos de muestreo adicionales en barras de vegetación media. En cada punto de muestreo se tomaron dos muestras alteradas para el análisis físicoquímico y dos muestras no alteradas con anillos de $100 \mathrm{~cm}^{3}$ para el cálculo de la densidad aparente (a 0-5 y $5-10 \mathrm{~cm}$ de profundidad en ambos casos). En total se dispuso de 60 puntos de muestreo de sedimentos y 121 puntos de muestreo de suelos distribuidos por los usos del suelo y depósitos sedimentarios (figura 1). Las muestras de suelo alteradas se secaron al aire, se tamizaron a $2 \mathrm{~mm}$ y se molieron para las determinaciones analíticas. Las muestras de suelo no alteradas se secaron a $105^{\circ} \mathrm{C}$, durante 24 horas, para la determinación de la densidad aparente. La densidad aparente fue corregida por la pedregosidad $>2 \mathrm{~mm}$, estimando su volumen y corrigiendo así el volumen del anillo (cuadro 1). El contenido de $\mathrm{CO}$ se determinó por combustión seca en un analizador elemental (FLASH EA 1112 SERIES Thermo) (cuadro 1). Estos valores medios de $\mathrm{CO}$ se utilizaron como base para calcular la existencia y el reservorio de $\mathrm{CO}$. La existencia de $\mathrm{CO}$ se estimó por medio de la ecuación 2:

$$
\mathrm{CO}_{\text {existencia }}=\mathrm{Da} * \mathrm{CO}^{\mathrm{i}} * \mathrm{P}
$$

Donde, $\mathrm{CO}_{\text {existencia }}=$ existencia de carbono orgánico $\left(\mathrm{Mg} \mathrm{ha}^{-1}\right)$. $\mathrm{Da}=$ densidad aparente $\left(\mathrm{g} \mathrm{cm}^{-3}\right) \cdot \mathrm{CO}^{\mathrm{i}}=$ contenido de carbono orgánico $(\%) . \mathrm{P}=$ profundidad de muestreo $(\mathrm{cm})$.

Para la estimación de la existencia de $\mathrm{CO}$ en los diferentes puntos de muestreo se agregaron los datos a una profundidad de $10 \mathrm{~cm}$, tal y cómo se ha utilizado en otros estudios de erosión-sedimentación de $\mathrm{CO}$ a escalas de cuenca y regionales (Chappell et al. 2014). La existencia de CO se combinó con la información espacial (patrón de usos del suelo y depósitos sedimentarios en 1956 y 2015) siguiendo la ecuación 3. Se determinó el reservorio de CO total $(\mathrm{Mg})$ (ecuación 3) y la existencia total a escala de cuenca y cauce $\left(\mathrm{Mg} \mathrm{ha}^{-1}\right)$. La existencia total resultó de dividir el reservorio total entre la superficie de la cuenca y la superficie del entorno fluvial (cauce y formas geomorfológicas) en cada año. Esta aproximación es equivalente a la seguida por Boix-Fayos et al. (2015) y Muñoz-Rojas et al. (2012). Para calcular el reservorio de CO en cada tipología espacial se utilizó la ecuación 3, siendo el reservorio total el sumatorio de todos ellos de acuerdo a la ecuación 4:

$$
\mathrm{CO}_{\text {reservorio_i }}=\left(\mathrm{CO}_{\text {existencia }} * \mathrm{X}_{\mathrm{i}}\right)
$$

Donde,

$\mathrm{CO}_{\text {reservorio } \mathrm{i}}=$ reservorio de $\mathrm{CO}(\mathrm{Mg})$.

$\mathrm{i}=$ uso de suelo específico.

$\mathrm{Xi}$ = área de un determinado uso de suelo o depósito sedimentario (ha).

$$
\mathrm{CO}_{\text {reservorio_total }}=\Sigma\left(\mathrm{CO}_{\text {existencia }} * \mathrm{X}_{\mathrm{i}}\right)
$$


Cuadro 1. Medias y desviación estándar del contenido de carbono orgánico (CO) y densidad aparente (DA) en distintos depósitos fluviales y suelos a $10 \mathrm{~cm}$ de profundidad [(Fuentes: Muestreo propio realizado en este trabajo junto a datos de Boix-Fayos et al. (2015) y Nadeu et al. $(2012,2013,2014,2015)]$.

Means and standard deviation of organic carbon content (CO) and bulk density (DA) at different fluvial deposits and soil at $10 \mathrm{~cm}$ depth (Own sampling for this work, in addition to Boix-Fayos et al. 2009, 2015, 2017, Nadeu et al. 2012, 2013, 2014, 2015).

\begin{tabular}{lccccc}
\hline \multicolumn{1}{c}{ Ámbitos de muestreo } & $\mathrm{n}$ & $\mathrm{DA}\left(\mathrm{g} / \mathrm{cm}^{3}\right)$ & $\sigma \mathrm{DA}$ & $\mathrm{CO}(\%)$ & $\sigma \mathrm{CO}$ \\
\hline Barra vegetada escasa & 8 & 1,3 & $\pm 1,3$ & 0,4 & $\pm 0,5$ \\
Barra vegetada media & 9 & 1,2 & $\pm 1,2$ & 0,4 & $\pm 0,1$ \\
Barra vegetada densa & 19 & 1,2 & $\pm 0,1$ & 0,8 & $\pm 0,1$ \\
Abanicos aluviales & 8 & 1,3 & $\pm 1,3$ & 0,6 & $\pm 0,1$ \\
Llanuras aluviales & 5 & 1,2 & $\pm 1,2$ & 0,4 & $\pm 0,1$ \\
Erosión lateral & 11 & 1,3 & $\pm 1,3$ & 0,5 & $\pm 0,1$ \\
Uso agrícola & 22 & 1,2 & $\pm 0,2$ & 1,0 & $\pm 0,3$ \\
Bosque abierto & 37 & 1,1 & $\pm 0,2$ & 2,8 & $\pm 1,5$ \\
Bosque denso & 23 & 1,0 & $\pm 0,2$ & 3,2 & $\pm 1,3$ \\
Matorral & 25 & 1,2 & $\pm 0,2$ & 1,7 & $\pm 0,8$ \\
Pasto & 14 & 1,3 & $\pm 0,2$ & 1,5 & $\pm 0,7$ \\
\hline
\end{tabular}

Donde,

$\mathrm{CO}_{\text {reservorio total }}=$ sumatorio del producto del $\mathrm{CO}_{\text {existencia }}$ por la superficie de todos los ámbitos de estudio, en los sedimentos fluviales o en los suelos de la cuenca.

\section{RESULTADOS}

Reverdecimiento en la cuenca de drenaje. La cobertura del suelo se modificó considerablemente entre 1956 y 2015 , principalmente el uso forestal, el cual aumentó 3.528 ha $(280 \%)$, mientras que los usos agrícola y matorral disminuyeron la superficie en 2.168 ha y 1.426 ha, el $-58 \%$ y $-63 \%$, respectivamente. El pasto, pese a aumentar la superficie en 142 ha, experimentó un cambio del $57 \%$; y el bosque abierto se mantuvo, perdiendo 126 ha, lo que supuso un cambio del $-4 \%$ (cuadro 2, figura 2). De acuerdo a los cambios mostrados en Pérez-Cutillas et al. (2018) en la cuenca del Alto Taibilla, se aprecia una evidente transición del uso del suelo predominante en la cuenca, desde el uso agrícola al uso forestal. Se observa el aumento de la densidad forestal al Este y Sur de la cuenca, donde en 1956 el uso predominante era el bosque abierto y cambió al bosque denso; aunque también se observa la transición desde el matorral al bosque denso en el centro y Oeste de la cuenca. La desaparición de las parcelas agrícolas se aprecia a lo largo de toda la cuenca, especialmente al Norte.

Transformaciones geomorfológicas. Entre 1956 y 2015 la longitud de cauce del río Turrilla disminuyó $0,5 \mathrm{~km}$, y la anchura media y la superficie del cauce activo se redujo el $63 \%$ en ambos casos, aunque la transformación varía según los tramos del cauce. En el tramo superior, la lon- gitud aumentó ligeramente, mientras que la superficie y la anchura del cauce activo disminuyeron un $63 \%$ y un $60 \%$, respectivamente. En el tramo intermedio la longitud aumentó de nuevo escasamente, al tiempo que tanto la superficie como la anchura decrecieron el $51 \%$. En el tramo inferior, a diferencia de los anteriores, la longitud del cauce activo se redujo alrededor de $0,6 \mathrm{~km}$, mientras que la superficie y la anchura se redujeron el $65 \%$ (figura 3 ). Además, en ese período, el conjunto de formas geomorfológicas identificadas aumentó el $14 \%$ en superficie y el $77 \%$ en número, pero los diferentes tipos no evolucionaron de forma homogénea. Las formas de erosión fueron muy ac-

Cuadro 2. Distribución de los usos del suelo en la cuenca del río Turrilla en 1956 y 2015 y el cambio observado en ha y proporcional (\%).

Land use cover of Turrilla Basin in 1956 and 2015 and the change observed in ha and proportional (\%).

\begin{tabular}{lcrrc}
\hline \multicolumn{1}{c}{ Tipo de uso } & 1956 & 2015 & $\begin{array}{c}\text { Cambio } \\
\text { (ha) }\end{array}$ & $\begin{array}{c}\text { Cambio } \\
(\%)\end{array}$ \\
\hline Uso agrícola & 3.765 & 1.643 & -2.122 & -58 \\
Bosque abierto & 3.135 & 3.009 & -126 & -4 \\
Bosque denso & 1.260 & 4.788 & 3.528 & 280 \\
Matorral & 2.272 & 846 & -1.426 & -63 \\
Pasto & 248 & 390 & 142 & 57 \\
Embalse del Taibilla & - & 3 & 3 & - \\
\hline Total & 10.680 & 10.680 & 0 & - \\
\hline
\end{tabular}




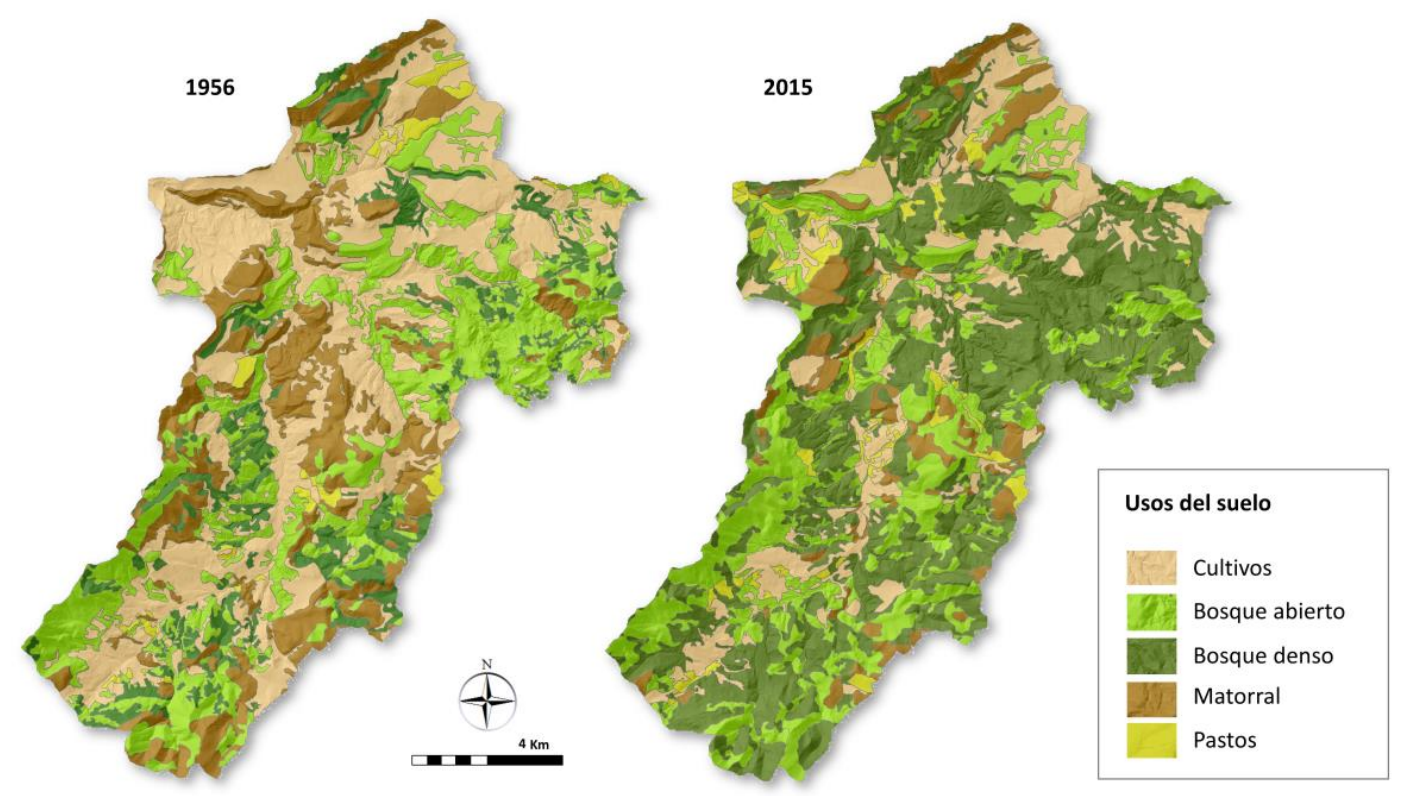

Figura 2. Cobertura del suelo de la cuenca del río Turrilla en 1956 y 2015.

Maps of land use cover in Turrilla Basin in 1956 and 2015.

tivas: la erosión lateral aumentó en superficie el $34 \%$, y el $59 \%$ en número de formas, lo cual encaja en una dinámica fluvial con alta capacidad de carga y con la incisión del cauce sobre el lecho; y la erosión en cárcavas aumentó en superficie y número el $21 \%$ y $24 \%$, respectivamente. De otra parte, las formas sedimentarias se ampliaron a medida que el cauce activo se estrechaba: la superficie de los abanicos aluviales aumentó el $13 \%$ sin modificarse el número de formas identificadas; y la superficie de las llanuras aluviales aumentó el 7 \% y el número de formas aumentó un $65 \%$, de acuerdo a un escenario con incisión del cauce (figura 3).

La transformación de las formas geomorfológicas todavía fue más heterogénea según el tramo del cauce dónde se localizaron: i) en el tramo superior el número de formas aumentó en las llanuras aluviales (92 \%) y en la erosión lateral (82\%). Respecto a la superficie ocupada, apareció una disparidad entre las formas de erosión que aumentaron en torno al $70 \%$ y las formas sedimentarias que lo hacen en torno al $20 \%$; ii) en el tramo intermedio la transformación del número de formas es similar en la erosión en cárcavas y en las llanuras aluviales (60\%) mientras que la erosión lateral aumenta más $(95 \%)$ y los abanicos aluviales se mantienen. En cuanto a la superficie, destaca la erosión en cárcavas (60\%); y, por último, iii) la transformación es parecida en el tramo inferior para todas las formas geomorfológicas, aunque en aumento de superficie destaca la erosión lateral (30\%), y en aumento del número de formas las llanuras aluviales (37\%). En todos los tramos las formas de erosión aumentan más en superficie que las sedimentarias (figura 4).
Reverdecimiento y estabilización del cauce del río Turrilla. El cauce experimentó un importante reverdecimiento entre 1956-2015: las barras fluviales vegetadas densamente (cobertura vegetal $\geq 70 \%$ ) aumentaron el $841 \%$; las barras con vegetación media (cobertura $>30 \mathrm{y}<70 \%$ ) aumentaron el $368 \%$; y el sedimento fluvial poco vegetado (cobertura $\leq 30 \%$ ) se redujo un $80 \%$. El reverdecimiento de estas formas todavía fue más evidente analizando los tramos del cauce. Por otra parte, tanto el trazado del curso fluvial como el número de cauces sobre el lecho fluvial variaron. Los resultados apuntaron hacia un cauce más estable en 2015. El índice braided, con una reducción de 1,19 (2,65 en 1956, 1,46 en 2015), mostró una estabilización considerable del cauce principal sobre los cauces secundarios, debido a la incisión de este en el lecho. El índice de sinuosidad, que analiza la morfología del trazado de flujo, disminuyó de 1,28 a 1,24 lo cual concuerda con la tendencia hacia un trazado rectilíneo y de acortamiento del cauce.

Evolución del reservorio de carbono orgánico en la cuenca del río Turrilla. El reservorio de carbono orgánico (CO) se cuantificó tanto en los diferentes usos del suelo en la cuenca de drenaje, como en el sedimento fluvial del cauce y en las formas geomorfológicas adyacentes al mismo. Los datos de muestreo se extrapolaron utilizando las superficies cartografiadas, en la cuenca y cauce, en 1956 y 2015 (cuadros 3 y 4). El sedimento fluvial contiene más CO cuanto más estable se encuentra el depósito y con mayor cobertura vegetal. El cuadro 3 muestra cómo las barras con cobertura vegetal densa aumentaron su reservorio de $\mathrm{CO}$ el $841 \%$, y con cobertura media el $368 \%$. Ambos tipos de 
barra fluvial albergaban el $57 \%$ del $\mathrm{CO}$ en el sedimento fluvial en 2015. En cambio, en las barras con cobertura escasa el reservorio de $\mathrm{CO}$ disminuyó el $80 \%$ comparado con 1956, debido a que éstas desaparecieron en una gran parte y evolucionaron hacia barras con cobertura media o densa (figura 5). Estos resultados concuerdan con el reverdecimiento del cauce observado en este período (figura 3). Por su parte, el reservorio de $\mathrm{CO}$ en los depósitos de las formas geomorfológicas adyacentes también se incrementó, aunque de forma más moderada. Las llanuras aluviales aumentaron un $7 \%$ el reservorio de $\mathrm{CO}$ en 2015 , representando el $23 \%$ del CO total de los sedimentos. Los abanicos aumentaron su reservorio un $14 \%$, y los depósitos generados por erosión lateral un $34 \%$, aunque ambas formas suponen menos del $10 \%$ del $\mathrm{CO}$ en el entorno fluvial en 2015. Con esto, el reservorio total de CO del conjunto de

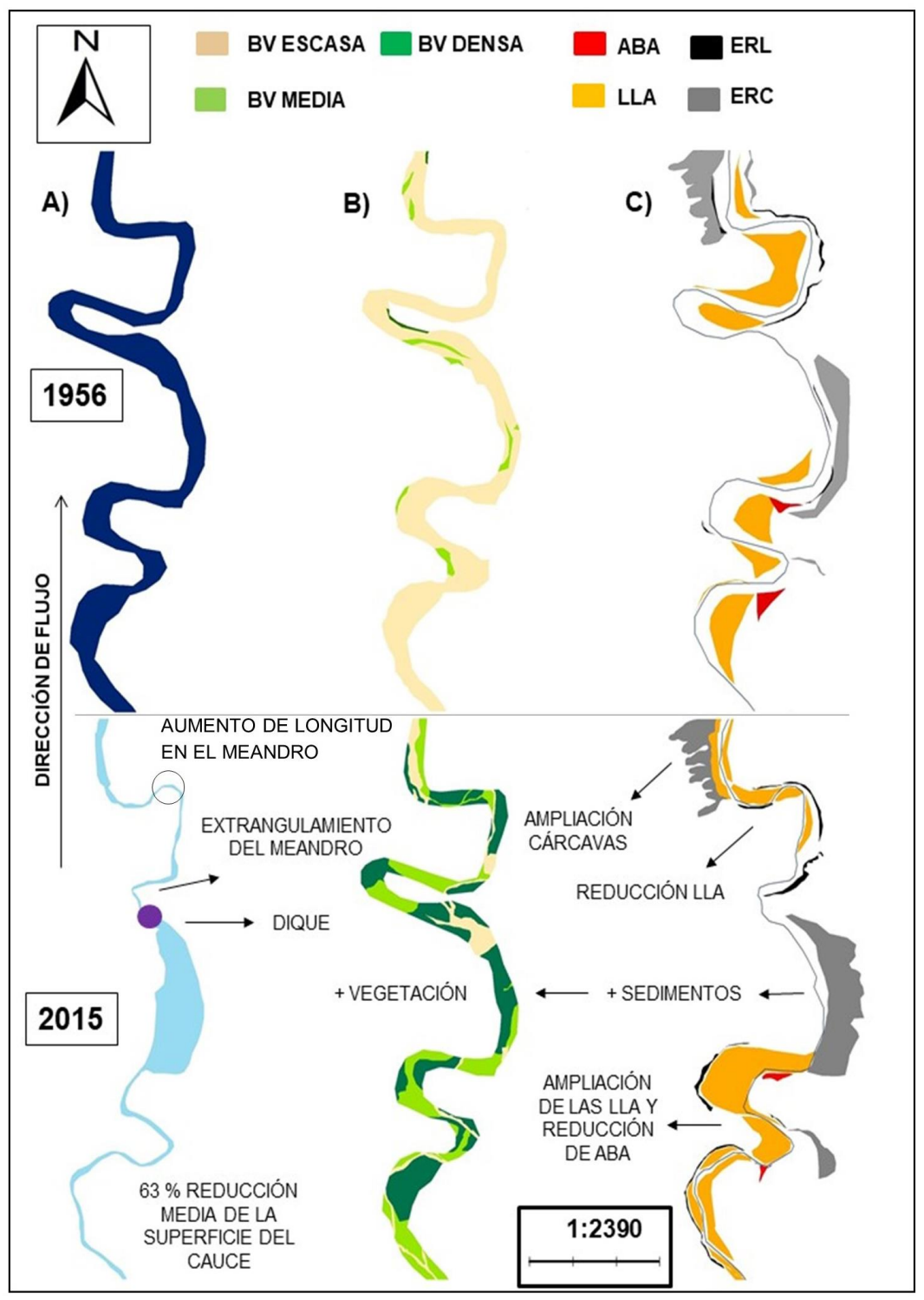

Figura 3. Análisis integral de las transformaciones en un sector del tramo intermedio del cauce: A) la superficie del cauce en 1956 y 2015 tras la instalación de un dique; B) la transformación de la cobertura vegetal en el cauce de 1956 transcurridos 59 años; y C) la transformación de las formas geomorfológicas durante 1956-2015. Representación de los tres tipos de cobertura vegetal y formas geomorfológicas analizadas: barras fluviales (BV), llanuras aluviales (LLA), abanicos aluviales (ABA), y procesos de erosión lateral (ERL) y erosión en cárcavas (ERC).

Analysis of the changes in a sector of the 2nd section of the channel: A) the surface of the channel in 1956 and 2015 after the construction of a check-dam; B) the change of the vegetal cover in the channel since 1956; and C) the change of geomorphological forms during 1956-2015. Representation of the three types of vegetation cover and geomorphological forms analyzed: type of fluvial bars (BV), alluvial plains (ALL), alluvial fans (ABA), bank erosion processes (ERL) and gully erosion (ERC). 
sedimentos fluviales aumentó $81 \mathrm{Mg}$, lo que supone un incremento del $27 \%$ respecto al reservorio de 1956 (cuadros 3 y 4), siendo este aumento paralelo a un aumento de la superficie del entorno fluvial, del cauce y las formas geomorfológicas, del 3,8\% debido al ampliación de las últimas.
Paralelamente, el reservorio de $\mathrm{CO}$ en los suelos de la cuenca de drenaje mostró un aumento considerable debido al aumento de la masa forestal (Pérez-Cutillas et al. 2018). El cuadro 3 también recoge estas transformaciones en el mismo período, en la que se observa que el bosque denso

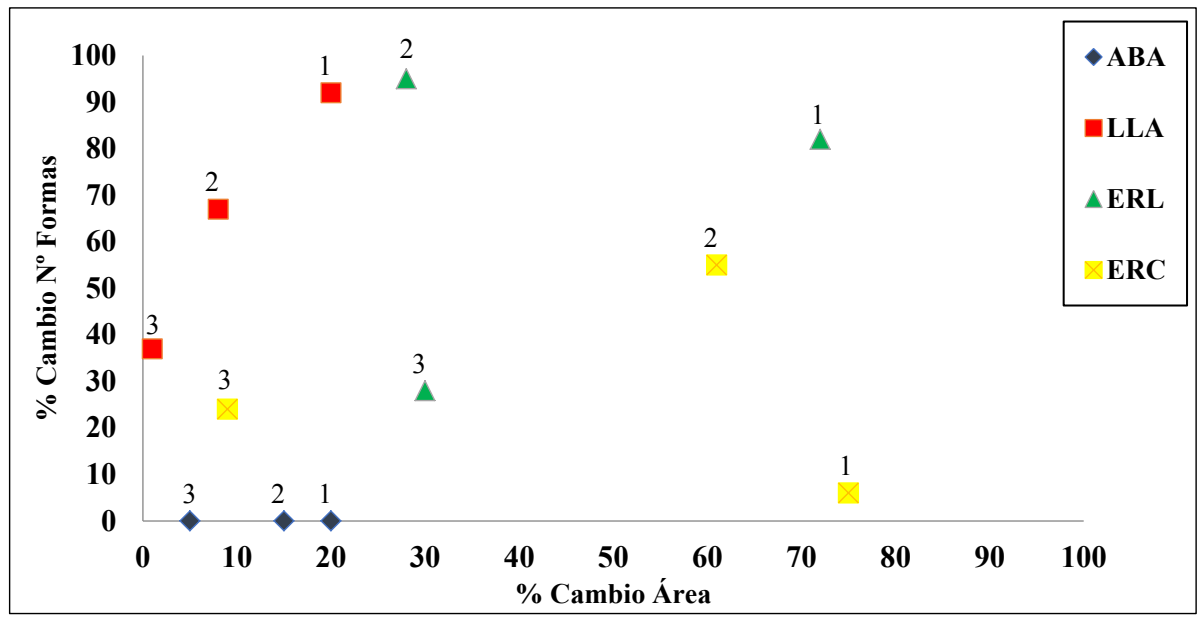

Figura 4. Transformación proporcional (\%) del área y número de formas de las FG entre 1956 y 2015. Se representa la transformación de los abanicos aluviales (ABA), las llanuras aluviales (LLA), la erosión lateral (ERL), y la erosión en cárcavas (ERC) en los diferentes tramos del cauce: superior (1), intermedio (2) e inferior (3).

Proportional changes (\%) of the area and number of geomorphological forms during 1956 and 2015. The transformation of alluvial fans (ABA), alluvial plains (ALL), bank erosion (ERL), and gullies (ERC) are represented in the different sections of the channel: high (1), second (2) and final (3).

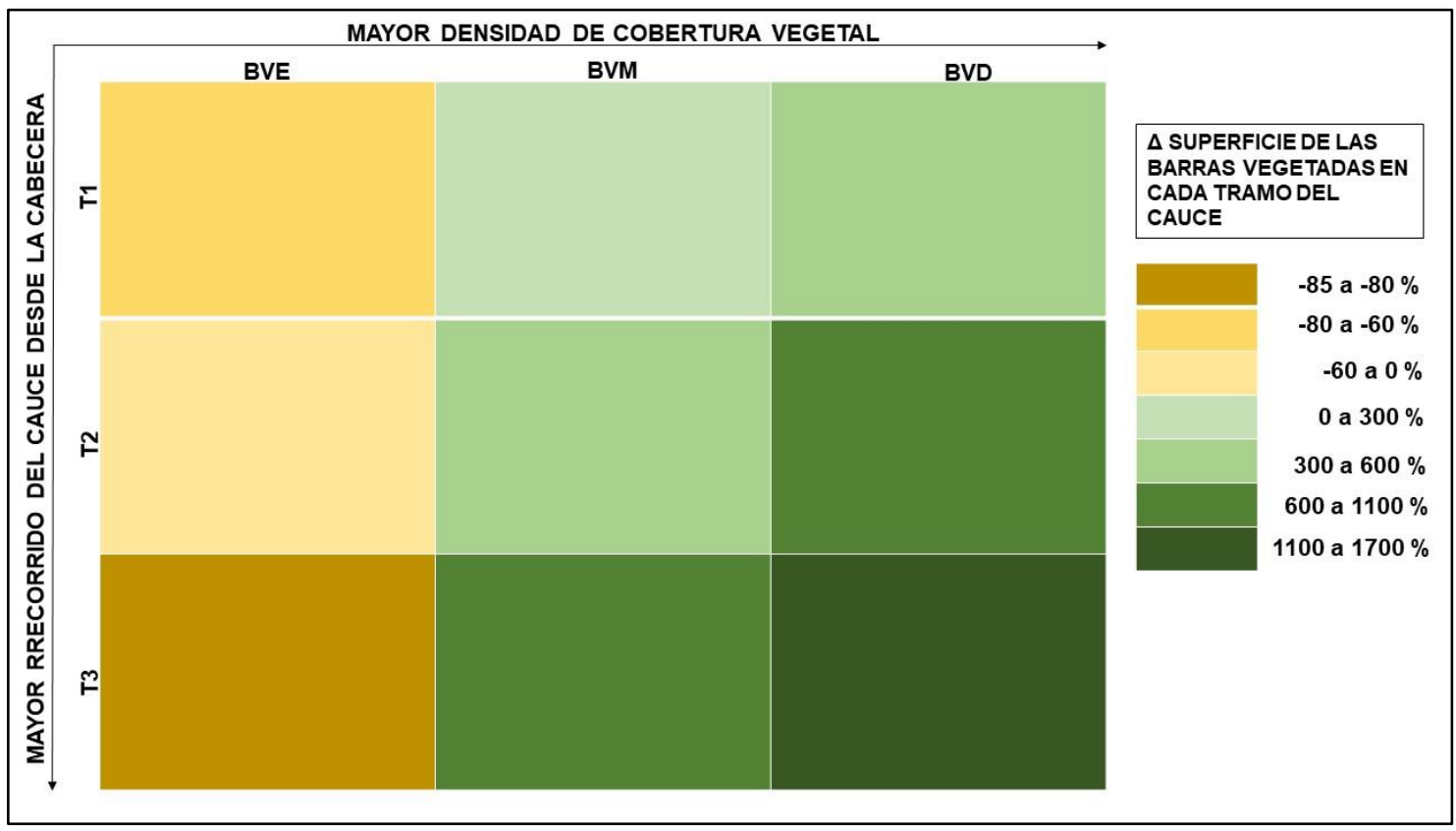

Figura 5. Transformación de las barras fluviales según densidad de cobertura vegetal y el tramo del río (BVE: Barra de vegetación escasa; BVM. Barra de vegetación media; BVD: Barra de vegetación densa).

Proportional changes (\%) of fluvial bars according to vegetation cover density and river section during 1956 and 2015: low vegetation bar (BVE), medium vegetation bar (BVM) and dense vegetation bar (BVD). 
Cuadro 3. Reservorio de $\mathrm{CO}$ en toneladas $(\mathrm{Mg})$ y porcentajes (\%) de cada ámbito espacial de muestreo en 1956 y 2015 , y la transformación durante ese período, en toneladas y en porcentaje.

Stocks of organic carbon (CO) in tons (Mg) and percentages (\%) in each spatial area of sampling in 1956 and 2015, and changes during that period, in tons and percentage. $(*)$ : There were no irrigated crops in 1956.

\begin{tabular}{lrrrrrr}
\hline \multirow{2}{*}{ Ámbitos de muestreo } & \multicolumn{2}{c}{ Reserva CO $(\mathrm{Mg})$} & \multicolumn{2}{c}{ \% Reserva $\mathrm{CO}$} & \multicolumn{2}{c}{ Cambio } \\
\cline { 2 - 7 } & \multicolumn{1}{c}{1956} & 2015 & 1956 & 2015 & $(\mathrm{Mg})$ & $(\%)$ \\
\hline Barra Vegetada Escasa & 146 & 29 & 49 & 8 & -116 & -80 \\
Barra Vegetada Media & 14 & 64 & 5 & 17 & 51 & 368 \\
Barra Vegetada Densa & 16 & 150 & 5 & 40 & 134 & 841 \\
Abanicos Aluviales & 31 & 35 & 10 & 9 & 4 & 14 \\
Llanuras Aluviales & 80 & 85 & 27 & 23 & 6 & 7 \\
Depósitos de erosión Lateral & 9 & 11 & 3 & 3 & 3 & 34 \\
\hline Total & 294 & 375 & 100 & 100 & 81 & 27 \\
\hline Cultivo de Regadío & - & 552 & 1 & - & 552 & - \\
Cultivo de Secano & 45.177 & 19.161 & 19 & 7 & -26.017 & -58 \\
Bosque Abierto & 96.554 & 92.692 & 41 & 32 & -3.862 & -4 \\
Bosque Denso & 40.324 & 153.210 & 17 & 53 & 112.886 & 280 \\
Matorral & 46.345 & 17.270 & 20 & 6 & -29.076 & -63 \\
Pasto & 4.841 & 7.605 & 2 & 3 & 2.764 & 57 \\
\hline Total & 233.241 & 290.489 & 100 & 100 & 57.247 & 25 \\
\hline
\end{tabular}

Cuadro 4. Reservorio total de $\mathrm{CO}(\mathrm{Mg})$, existencia de $\mathrm{CO}(\mathrm{Mg}$ $\mathrm{ha}^{-1}$ ) y tasa de variación (TV) en el sedimento fluvial y en los suelos de la cuenca en los años 1956 y 2015.

Reservoir of organic carbon (CO) (total value, $\mathrm{Mg}$ ) and stock $\left(\mathrm{Mg} \mathrm{ha}^{-1}\right)$ and variation rate $(\mathrm{TV} *)$ in the fluvial sediment and the soil of the basin in 1956 and 2015 .

\begin{tabular}{|c|c|c|c|}
\hline \multicolumn{4}{|c|}{ Sedimento fluvial (Barras fluviales y FG) } \\
\hline & 1956 & 2015 & $\begin{array}{c}\text { Cambio } \\
(\%)\end{array}$ \\
\hline Superficie (ha) & 56 & 58 & 4 \\
\hline Reservorio total de $\mathrm{CO}(\mathrm{Mg})$ & 294 & 375 & 27 \\
\hline Existencia de $\mathrm{CO}\left(\mathrm{Mg} \mathrm{ha}^{-1}\right)$ & 5 & 6 & 23 \\
\hline \multicolumn{4}{|c|}{ Suelos de la cuenca (Usos) } \\
\hline & 1956 & 2015 & $\begin{array}{c}\text { Cambio } \\
(\%)\end{array}$ \\
\hline Superficie (ha) & 10.690 & 10.690 & - \\
\hline Reservorio total de $\mathrm{CO}(\mathrm{Mg})$ & 233.241 & 290.489 & 25 \\
\hline Existencia de $\mathrm{CO}\left(\mathrm{Mg} \mathrm{ha}^{-1}\right)$ & 22 & 27 & 25 \\
\hline \multicolumn{4}{|c|}{ Ratio reservorio CO (Sedimentos/Suelos) } \\
\hline & 1956 & 2015 & \\
\hline $\begin{array}{l}\text { Ratio de reservorio total de } \\
\text { CO sedimentos/suelo }\end{array}$ & 0,0013 & 0,0013 & \\
\hline $\begin{array}{l}\text { Ratio de existencia de CO } \\
\text { sedimentos/suelo }\end{array}$ & 0,24 & 0,24 & \\
\hline
\end{tabular}

incrementó su reservorio de $\mathrm{CO}$ el $280 \%$, el único uso del suelo con incremento junto a los pastos (46\%). Además, el bosque denso junto al bosque abierto albergó el $92 \%$ del CO total en 2015. En el lado opuesto, el descenso de la superficie agrícola propició la disminución del reservorio de CO (-58 \%). Algo similar sucedió con el bosque abierto debido a que se transformó a bosque denso (-4 \%). En el matorral el reservorio de $\mathrm{CO}$ descendió el $63 \%$, de acuerdo al decrecimiento de su extensión. En el balance global, el CO en la cuenca de drenaje aumentó $57.247 \mathrm{Mg}$, lo que supuso un incremento del $25 \%$ respecto a 1956 (cuadros 3 y 4 ).

El cuadro 4 muestra los cambios en el reservorio total y la existencia de $\mathrm{CO}$ en los suelos de la cuenca y en el sedimento fluvial durante el período de estudio. Mientras que la superficie de la cuenca no cambió en el tiempo, el cauce activo se estrechó y los depósitos fluviales dentro del cauce aumentaron su extensión (figura 3). El reservorio total de CO aumentó un $25 \%$ en los suelos de la cuenca, con el mismo aumento por unidad espacial, del $25 \%$ en la existencia. En los depósitos de sedimentos del cauce y formas geomorfológicas adyacentes, el reservorio total de $\mathrm{CO}$ aumentó el $27 \%$, al revegetarse el cauce, extenderse las barras fluviales con cobertura vegetal densa y aumentar la extensión de las formas sedimentarias. Sin embargo, la existencia aumenta el $23 \%$. Así observamos un aumento muy similar del reservorio total de $\mathrm{CO}$ en los sedimentos y en los suelos (27\% y $25 \%$, respectivamente). Así como 
el aumento de la existencia muy similar en sedimentos y suelos ( $23 \%$ y $25 \%$, respectivamente).

La razón entre el reservorio total de $\mathrm{CO}$ de los sedimentos y la de los suelos es muy baja, teniendo en cuenta que el cauce representa aproximadamente solo un $0,5 \%$ de la superficie de la cuenca. Las razones entre $\mathrm{CO}$ en sedimentos y suelos tanto del reservorio total como de la existencia se mantienen a los mismos niveles en las dos fechas estudiadas (0,24 para 1956 y 2015). Mientras que en las dos fechas el CO almacenado en el cauce también se mantiene en un $0,13 \%$ del $\mathrm{CO}$ almacenado en los suelos de la cuenca (cuadro 4).

\section{DISCUSIÓN}

Cambios principales en la morfología del cance debido a modificaciones en la cuenca de drenaje. El patrón de usos del suelo en la cuenca del río Turrilla varió notablemente durante los últimos 60 años como consecuencia del abandono progresivo de tierras agrícolas que favoreció la regeneración natural de la vegetación, así como por la implantación de políticas ambientales de restauración hidrológico-forestal (Pérez-Cutillas et al. 2018). De este modo, la cuenca experimentó un reverdecimiento significativo que coincide con lo observado durante las últimas décadas en España (Vadell et al. 2016), en otras cuencas mediterráneas de montaña (García-Ruíz y Lana-Renault 2011), así como en otras zonas del planeta (Wei et al. 2012, Sun-Kim et al. 2017). Como consecuencia, la estabilidad de los suelos aumentó, y con ello disminuyeron los procesos de erosión en laderas y la conectividad ladera-cauce (Boix-Fayos et al. 2007), lo que afectó a la dinámica sedimentaria en el conjunto de la cuenca, con una menor movilización de sedimentos en el cauce con respecto a lo observado en la década de 1950. Además de los impactos humanos en el área de drenaje, en la década de 1970 se alteraron un gran número de cauces, con la instalación de diques para laminación de avenidas y lucha contra la erosión (GómezVillar y Martínez-Castroviejo 1991). Concretamente se contabilizaron 58 diques (Boix-Fayos et al. 2007) en la rambla de la Rogativa (subcuenca afluente del río Turrilla), que implicaron evidentes transformaciones sobre la dinámica fluvial, alterando la distribución de los sedimentos en el cauce. En muchas ocasiones, los diques en el cauce generaron una mayor capacidad de carga del caudal aguas abajo de los mismos, debido a la retención de sedimentos aguas arriba tras la pared del dique, y, en numerosas ocasiones, a largo plazo esto derivó en procesos de erosión del lecho y erosión lateral (Kondolf et al. 2002, Boix-Fayos et al. 2007, Nadeu et al. 2012).

Todos estos procesos descritos se reflejan en las transformaciones morfológicas observadas en el cauce: i) tanto la superficie como la anchura del mismo se redujeron el $63 \%$ en el período estudiado, ii) la longitud total del mismo también disminuyó $(0,5 \mathrm{~km})$, aunque no fue así en los tramos intermedio y superior, debido a la presencia de nume- rosos diques que propiciaron la incisión del cauce aguas abajo, provocando que los meandros se extendieran en su margen de erosión (figura 4). Por su parte, la incisión y estrechamiento del cauce permitió la estabilización de los depósitos sedimentarios, y la colonización de la vegetación en los mismos (figura 4). Estos procesos son similares a los descritos en otras zonas (Kondolf et al. 2002, Stott y Mount 2004, Vanacker et al. 2005).

Además, los indicadores de la estabilidad del cauce empleados, el índice braided y el índice de sinuosidad, mostraron una tendencia hacia una mayor estabilidad durante el periodo de estudio. Los cambios de ambos índices implican una mayor longitud del cauce principal sobre la longitud de los cauces secundarios y una ligera tendencia hacia el trazado rectilíneo del cauce, respectivamente (Schumm 1963, Wei-Kuo et al. 2017).

De otra parte, se cuantificó un notable reverdecimiento de la superficie del cauce activo comparado con 1956 (figuras 3 y 6), con una ampliación considerable de la superficie de barras fluviales con cobertura vegetal densa y media (figura 5). En este escenario de dinámica fluvial, la superficie de depósitos de sedimentos en las formas adyacentes al cauce aumentó (figuras 3, 4 y 6). Los abanicos y llanuras aluviales, ampliaron su superficie a medida que el cauce activo se estrechaba (figuras 3 y 4), así como la evolución de la llanura aluvial previa a terrazas fluviales al aparecer incisión fluvial, lo que favoreció la estabilización de las mismas por colonización vegetal (figura 6). La erosión en cárcavas aumentó en algunas zonas, sobre todo en el tramo medio, debido al aumento de la capacidad de carga. Ello supuso un importante aporte de sedimentos al cauce en dos ambientes principalmente: en algunas laderas limítrofes y bien conectadas con el cauce; y en barrancos afluentes con flujos frecuentes o con mucha pendiente. El aumento de la capacidad de carga supuso también el aumento de la erosión lateral $(>80 \%$ en los tramos superior y medio y $30 \%$ en el tramo inferior), con gran aporte de sedimentos al cauce, coincidiendo con observaciones realizadas previamente en algunos tramos y barrancos subsidiarios (Boix-Fayos et al. 2007, Nadeu et al. 2012).

Impactos de la dinámica sedimentaria en el reservorio de CO del cauce: ¿pueden ser los cauces un sumidero de carbono? La gestión del suelo para reducir la concentración de $\mathrm{CO}_{2}$ en la atmósfera aparece como una medida importante para la mitigación del cambio climático respaldada por la comunidad científica y las Naciones Unidas (Sanz-Cobena et al. 2017). Al mismo tiempo, todavía no se encuentran bien definidos los procesos que determinan el contenido de $\mathrm{CO}$ en el sedimento fluvial y su efecto en el ciclo global del carbono (Hoffman et al. 2013). Recientes investigaciones han señalado que la estabilización y reverdecimiento del sedimento fluvial puede suponer un importante aumento del reservorio de $\mathrm{CO}$ en las cuencas hidrológicas (Boix-Fayos et al. 2015), o, por el contrario, 


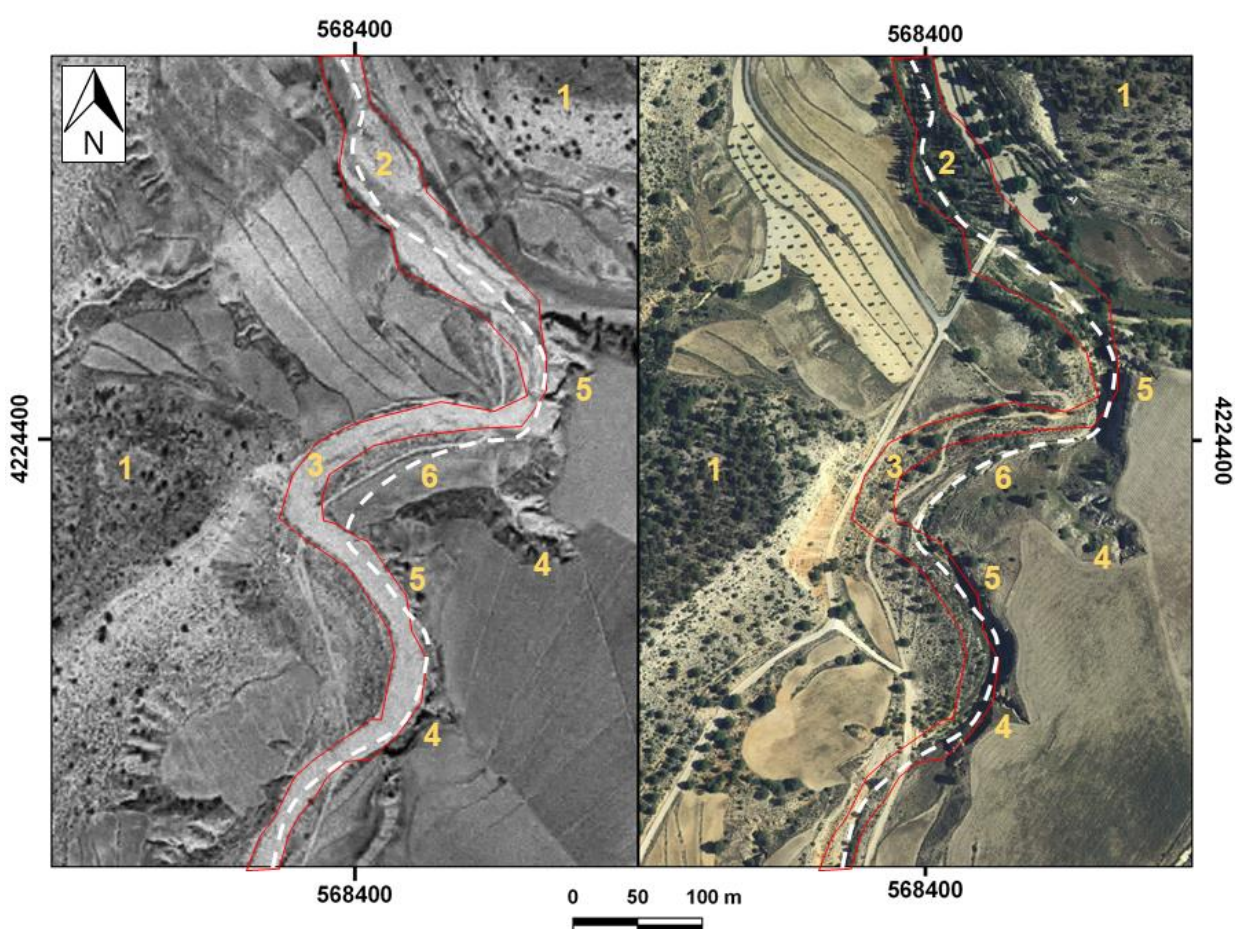

Figura 6. Ejemplos de cambios observados en la cuenca de drenaje y el cauce del río Turrilla (tramo inferior) mediante la imagen aérea de 1956 (izquierda) y 2015 (derecha): 1) revegetación de la cuenca y extensión del bosque denso; 2) aumento del bosque de ribera en el cauce, especialmente en el tramo inferior tras la confluencia con Arroyo Blanco; 3) revegetación del cauce activo de 1956; 4) erosión remontante por acarcavamiento; 5) aumento de la erosión lateral; y 6) abandono y revegetación de parcelas de cultivo tradicionales desde 1956.

Examples of changes observed in the drainage basin and the channel of Turrilla River (lower section) using the aerial image of 1956 (left) and 2015 (right): 1) revegetation of the basin and extension of the dense forest; 2) increase of the riparian forest in the channel, especially in the lower section after the confluence with Arroyo Blanco; 3) revegetation of the active channel of 1956; 4) increased erosion by gullies; 5) bank erosion processes; and 6) abandonment and revegetation of traditional farming fields since 1956.

dependiendo de los ambientes sedimentarios y los procesos que los afectan podrían aumentar el volumen de emisiones de $\mathrm{CO}_{2}$ a la atmósfera (Hoffman et al. 2013).

Los resultados en la cuenca del río Turrilla muestran que la cuenca de drenaje incrementó el reservorio de $\mathrm{CO}$ en los suelos en consonancia al aumento del suelo forestal, debido a la reducción de las actividades agrarias y a las políticas de reforestación. Todo ello facilitó la incorporación de $\mathrm{CO}$ al suelo (cuadro 3). Los cambios en la dinámica fluvial como consecuencia de los cambios en la cuenca drenaje, con la colonización vegetal de formas sedimentarias, extensión de la llanura aluvial en algunos tramos y estabilización y extensión de otros depósitos, llevaron a un aumento del reservorio total de carbono en el cauce, muy paralelo al aumento del reservorio total en los suelos de la cuenca (27\% y $25 \%$, respectivamente). Ello muestra el gran potencial de los sedimentos fluviales para generar sumideros de $\mathrm{CO}$ si se dan los procesos de revegetación y estabilización del sedimento fluvial. El aumento de la existencia de $\mathrm{CO}$ es similar en porcentaje en sedimentos y suelos (23 y $25 \%$, respectivamente). Sin embargo, en valores absolutos, el aumento fue de $1,2 \mathrm{Mg} \mathrm{ha}^{-1}$ en sedimentos y de 5,4 $\mathrm{Mg} \mathrm{ha}^{-1}$ en los suelos, reflejando la mayor capacidad de secuestro de carbono de los suelos, por su distintas características estructurales y texturales y por la vegetación existente. Aun así, los cambios en la morfología del cauce representaron una oportunidad para el aumento del sumidero de carbono en sedimentos, tanto por su extensión como por su estabilización. La existencia de $\mathrm{CO}\left(\mathrm{Mg} \mathrm{ha}^{-1}\right)$ en los sedimentos con respecto a los suelos se mantiene entre 1956 y 2015 (0,24 en ambos períodos) y coincide con observaciones realizadas a escala de cuenca de drenaje donde los sedimentos se encuentran empobrecidos en carbono orgánico en comparación con los suelos del área vertiente (Haregeweyn et al. 2007, Ran et al. 2014, BoixFayos et al. 2015). La razón entre la existencia de carbono en sedimentos respecto a los suelos se mantuvo en el tiempo en 0,24 mostrando la gran capacidad de los sedimentos de secuestrar carbono con los cambios acontecidos en la cuenca y cauce.

De este modo se observa: (i) una clara conexión entre el reverdecimiento de la cuenca y el reverdecimiento en el cauce (entorno fluvial), (ii) los sedimentos fluviales mostraron una capacidad similar a la de los suelos de la 


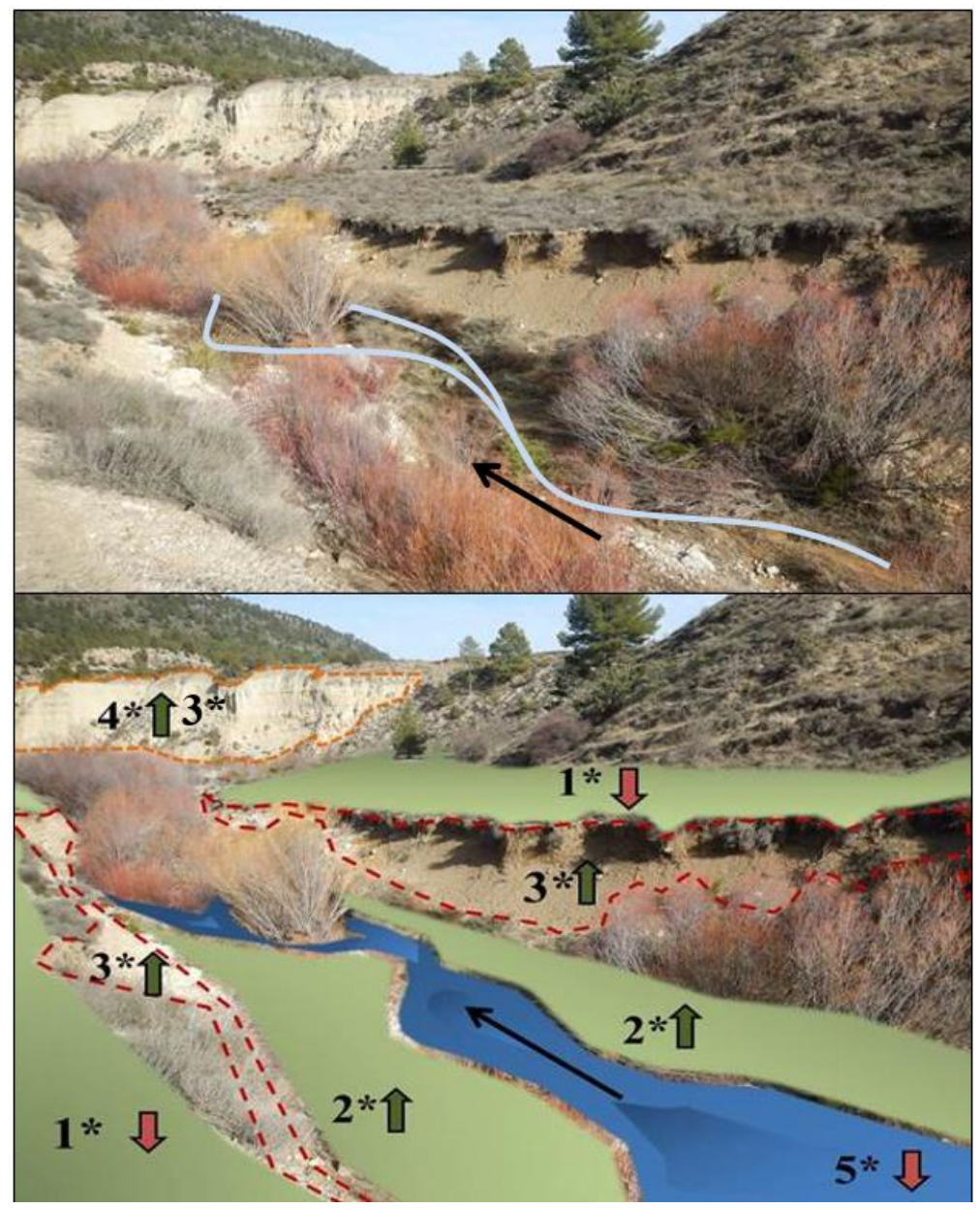

Figura 7. Se observa la actividad geomorfológica junto al cauce del río Turrilla, densamente vegetado. La llanura aluvial del cauce en $1956(1 *)$ disminuye por erosión lateral y se convierte en terraza fluvial, aparece una nueva llanura $(2 *)$. La erosión lateral ( $3 *)$ y la erosión en cárcavas $\left(4^{*}\right)$ se encuentran muy activas especialmente en el tramo medio e inferior. Las flechas verdes hacen referencia al aumento de la morfología señalada y las rojas al descenso de la misma entre los dos períodos estudiados.

The geomorphological activity is observed next to the fluvial channel of Turrilla, densely vegetated. The alluvial plains of the channel in $1956\left(1^{*}\right)$ decreased while current alluvial plains increased $(2 *)$, bank erosion $\left(3^{*}\right)$ and gullies $\left(4^{*}\right)$ are very active, especially in middle and lower sections. Green arrows refer to the increase and red arrows to the decrease of geomorphological forms during the study period.

cuenca para aumentar el reservorio total de $\mathrm{CO}$, lo que sugiere que los cauces con procesos de revegetación y reducción de la actividad hidrogeomorfológica tienen un gran potencial como sumideros de carbono; iii) las zonas sedimentarias una vez estabilizadas pueden convertirse en importantes sumideros de carbono, y aunque en extensión son mucho menores que el área de drenaje, el cauce y las morfologías adyacentes pueden suponer una trampa de sedimentos que ayudaría a mantener el carbono en el ecosistema fluvial. Además, la limitada extensión espacial puede compensarse con la profundidad de algunos depósitos sedimentarios (de varios metros). Aunque en este estudio se han comparado los 10 primeros centímetros tanto en sedimentos como en suelos, algunos depósitos sedimentarios de varios metros de profundidad (cuñas sedimentarias tras diques, depósitos de desplomes de erosión lateral, etc.) poseen un elevado potencial para el secuestro de carbono en profundidad.

Estos resultados indican que la gestión adecuada de sedimentos en los cauces, atendiendo a los factores y condiciones ambientales locales, puede aumentar el secuestro de carbono en los mismos. Los resultados coinciden con los de otros autores que apuntan el posible potencial de los sedimentos fluviales como sumideros de carbono (Hoffman et al. 2013), contribuyendo a disminuir el volumen de $\mathrm{CO}_{2}$ en la atmósfera, considerado una amenaza ambiental para el ser humano durante el presente siglo (Wei et al. 2012, Lal et al. 2015). Los resultados indican que una gestión sostenible de los sedimentos fluviales podría contribuir a las políticas de mitigación del cambio climático, de acuerdo a las proyecciones y recomendaciones del IPCC para el Siglo XXI (IPCC 2014). 


\section{CONCLUSIONES}

El proceso de reverdecimiento en la cuenca genera impactos en la dinámica fluvial, altera la entrada de sedimentos al cauce desde las laderas, propicia un estrechamiento del cauce activo, al tiempo que activa otros procesos de erosión (erosión lateral, cárcavas adyacentes) y estabiliza los depósitos fluviales (barras, llanuras y abanicos), que son colonizados parcial o totalmente por la vegetación.

El reservorio total de $\mathrm{CO}$ en suelos y sedimentos aumenta de forma paralela ( 27 y $25 \%$, respectivamente), el aumento de la existencia de $\mathrm{CO}$ en los sedimentos es bastante alto (23\%), a pesar del tiempo limitado de estabilización de los depósitos y del dinamismo que implica un ambiente geomorfológico fluvial. El aumento de la existencia de $\mathrm{CO}$ en suelos es similar (25\%). A pesar que la superficie del cauce es muy limitada $(-0,5-1 \%$ de la cuenca), la profundidad y estabilidad de algunos depósitos sedimentarios representan una oportunidad para el secuestro de carbono en las zonas de ribera.

Se confirma que los sedimentos fluviales estabilizados tras cambios en la dinámica geomorfológica, pueden suponer importantes sumideros de $\mathrm{CO}$, al igual que los suelos de las cuencas hidrológicas que experimentan recuperación de la cubierta vegetal. Con un manejo adecuado, tanto suelos como sedimentos, pueden contribuir significativamente a ampliar el sumidero de carbono en la biosfera terrestre.

\section{AGRADECIMIENTOS}

Este estudio ha recibido apoyo financiero del proyecto DISECO (CGL2014-55405-R) del Plan Nacional de Ciencia del Ministerio de Economía y Competitividad de España, del proyecto SOGLO (P7/24 IAP BELSPO) del gobierno de Bélgica. AHM recibió apoyo financiero para una estancia en la Universidad Nacional de Córdoba (Argentina) del Banco de Santander mediante el Convenio Becas de Intercambio Latinoamérica (Programa ILA). CBF recibió apoyo financiero para dos estancias en el extranjero del programa Salvador de Madariaga 2017 (Ministerio de Educación, Cultura y Deporte, Gobierno de España) y del programa Jiménez de la Espada 2017 (Fundación Séneca, Agencia de Ciencia y Tecnología de la Región de Murcia). MAB fue parcialmente financiada por un contrato Juan de la Cierva-Incorporación (Ref: IJCI-2015-23500). Todas estas estancias permitieron el trabajo continuado en la redacción de este artículo.

\section{REFERENCIAS}

AEMET e IM (Agencia Estatal de Meteorología, ES - Instituto de Meteorología, PT). 2011. Atlas Climático Ibérico: Temperatura del aire y precipitación durante 1971-2000. Consultado 17 jul. 2018. Disponible en http://agroclimap.aemet.es/

Boix-Fayos C, GG Barbera, F López-Bermúdez VM Castillo.
2007. Effects of check dams, reforestation and land-use changes on river channel morphology: Case study of the Rogativa catchment (Murcia, Spain). Geomorphology 91(1-2): 103-123. DOI: 10.1016/j.geomorph.2007.02.003

Boix-Fayos C, E Nadeu, JM Quiñonero, M Martínez-Mena, M Almagro, J de Vente. 2015. Sediment flow paths and associated organic carbon dynamics across a Mediterranean catchment. Hydrology and Earth System Sciences 19: 12091223. DOI: $10.5194 /$ hess-19-1209-2015

Chappell A, NP Webb, RA Viscarra Rossel, E Bui. 2014. Australian net (1950s-1990) soil organic carbon erosion: implications for $\mathrm{CO} 2$ emission and land-atmosphere modelling. Biogeosciences 11: 5235-5244. DOI: 10.5194/bg-11-5235-2014

García-Ruíz JM, N Lana-Renault. 2011. Hydrological and erosive consequences of farmland abandonment in Europe, with special reference to the Mediterranean region - A review. Agriculture, Ecosystems and Environments 140: 317-338. DOI: $\underline{10.1016 / \text { j.agee.2011.01.003 }}$

Gómez-Villar A, R Martínez-Castroviejo. 1991. Channel degradation as a response to erosion control Works: a case study. In Sala M, JL Rubio, JM García-Ruíz eds. Soil Erosion Studies in Spain. Logroño, Spain. Geoforma. p. 109-122.

Haregeweyn N, J Poesen, J Deckers, J Nyssen, M Hayle, G Govers, G Verstraeten, J Moeyersons. 2007. Sediment-bound nutrient export from micro-dam catchments in Northern Ethiopia. Land Degradation \& Development 19: 136-152. DOI: $10.1002 / \mathrm{ddr} .830$

Hoffmann T, SM Mudd, K van Oost, G Verstraeten, G Erkens, A Lang, H Middelkoop, J Boyle, JO Kaplan, J Willenbring, R Aalto. 2013. Short communication: Humans and the missing C-sink: erosion and burial of soil carbon through time. Earth Surface Dynamics 1: 45-52. DOI: 10.5194/ esurf-1-45-2013

Kondolf GM, H Piégay, N Landon. 2002. Channel response to increased and decrease bedload supply from land use change: contrasts between two catchments. Geomorphology 45: 35-51. https://doi.org/10.1016/S0169-555X(01)00188-X

IPCC (Intergovernmental Panel on Climate Change). 2014. Cambio climático 2014: contribución de los Grupos de trabajo I, II y III al Quinto Informe de Evaluación del Grupo Intergubernamental de Expertos sobre el Cambio Climático IPCC. 157 p. (Informe de síntesis).

Lal R, W Negassa, K Lorenz. 2015. Carbon sequestration in soil. Current Opinion in Environmental Sustainability. 15: 7986. DOI: 10.1016/j.cosust.2015.09.002

Muñoz-Rojas M, A Jordán, LM Zavala, D De la Rosa, SK AbdElmabod, M Anaya-Romero. 2012. Organic carbon stocks in Mediterranean soil types under different land uses. Solid Earth 3: 375-386. DOI: $10.5194 / \mathrm{se}-3-375-2012$

Nadeu E, AA Berhe, J De Vente, C Boix-Fayos. 2012. Erosion, deposition and replacement of soil organic carbon in Mediterranean catchments: a geomorphological, isotopic and land use change approach. Biogeosciences 9: 1099-1111. DOI: $10.5194 /$ bg-9-1099-2012

Nadeu E. 2013. Soil erosion and organic carbon mobilization at the catchment scale. Factors, processes and impact on the carbon balance. Consultado 10 jul. 2018. Disponible en: https://digitum.um.es/xmlui/handle/10201/30468

Nadeu E, K Van Oost, C Boix-Fayos, J de Vente. 2014. Importance of land use patterns for erosion-induced carbon fluxes in a Mediterranean catchment. Agriculture, Ecosystems \& En- 
vironment. 189: 181-189. DOI: 10.1016/j.agee.2014.03.040

Nadeu E, JM Quiñonero-Rubio, J de Vente, C Boix-Fayos. 2015. The influence of catchment morphology, lithology and land use on soil organic carbon export in a Mediterranean mountain region. Catena 126: 117-125. DOI: 10.1016/j.catena.2014.11.006

Pérez-Cutillas P, MF Cataldo, DA Zema, J de Vente, C BoixFayos. 2018. Efectos de la revegetación a escala de cuenca sobre el caudal y la evapotranspiración en ambiente mediterráneo. Cuenca del Taibilla (SE de España). Bosque 39(1): 119-129. DOI: $10.4067 /$ S0717-92002018000100011

Ran L, XX Lu, Z Xin. 2014. Erosion-induced massive organic carbon burial and carbon emission in the Yellow River basin, China. Biogeosciences 11: 945-959. DOI: 10.5194/bg11-945-2014

Sanz-Cobena A, L Lassaletta, J Garnier, P Smith. 2017. Mitigation and quantification of greenhouse gas emissions in Mediterranean cropping systems. Agriculture Ecosystems \& Environment 238: 1-4. DOI: 10.1016/j.agee.2016.12.032

Schumm SA. 1963. A tentative classification of alluvial river channels. Washington, EE.UU. Government Printing Office. 9 p.

Stott T, N Mount. 2004. Plantation forestry impacts on sediment yields and downstream channel dynamics in the UK: a re- view. Progress in Physical Geography 28: 197-240. DOI: $\underline{10.1191 / 0309133304 p p 410 \mathrm{ra}}$

Sun-Kim G, CH Lim, S Jin-Kim, J Lee, Y Son, WK Lee. 2017. Effect of National-Scale afforestation on forest water supply and soil loss in South Korea, 1971-2010. Sustainability 9(1017): 1-18. DOI: 10.3390/su9061017

Vadell E, S de-Miguel J Pernán. 2016. Large-scale reforestation and afforestation policy in Spain: a historical review of its underlying ecological, socioeconomic and political dynamics. Land Use Policy 55: 37-48. DOI: 10.1016/j.landusepol.2016.03.017

Vanacker V, A Molina, G Govers, J Poesen, G Dercon, S Deckers. 2005. River channel response to short-term human-induced change in landscape connectivity in Andean ecosystems. Geomorphology 72: 340-355. DOI: 10.1016/j.geomorph.2005.05.013

Wei-Kuo C, C Chi-Farn, C Su-Chin, Y Tun-Chi, C Chun-Wei. 2017. Channel Planform Dynamics monitoring and channel stability assessment in two sediment rich rivers in Taiwan. Water 9(2): 84. DOI: 10.3390/w9020084

Wei X, L Qiu, M Shao, X Zhang, W Gale. 2012. The accumulation of organic carbon in mineral soils by afforestation of abandoned farmland. PLoS ONE 7(3). DOI: $\underline{10.1371 / \text { jour- }}$ nal.pone. 0032054 